\title{
Adverse drug reaction and toxicity caused by commonly used antimicrobials in canine practice
}

\author{
K. Arunvikram ${ }^{1}$, Ipsita Mohanty ${ }^{2}$, K. K. Sardar ${ }^{2}$, S. Palai ${ }^{2}$, G. Sahoo ${ }^{3}$ and R. C. Patra ${ }^{4}$ \\ 1. Division of Pharmacology and Toxicology, Indian Veterinary Research Institute, Izatnagar -243122, Uttar Pradesh, \\ India: 2. Department of Pharmacology and Toxicology, Veterinary College, Orissa University of Agricultural and \\ Technology, Bhubaneswar - 751 003, Odisha, India; 3. Department of Biochemistry, Veterinary College, \\ Orissa University of Agricultural and Technology, Bhubaneswar - 751 003, Odisha, India; 4. Department of Clinical \\ Medicine, Veterinary College, Orissa University of Agricultural and Technology, Bhubaneswar - 751 003, Odisha, India \\ Corresponding author: Ipsita Mohanty, email: mohantyipsita8@gmail.com \\ Received: 20-03-2014, Revised: 05-04-2014, Accepted: 08-04-2014, Published online: 08-05-2014
}

doi: 10.14202/vetworld.2014.299-305

How to cite this article: Arunvikram K, Mohanty I, Sardar KK, Palai S, Sahoo G and Patra RC (2014) Adverse drug reaction and toxicity caused by commonly used antimicrobials in canine practice, Veterinary World 7(5): 299-305.

\begin{abstract}
An adverse drug reaction (ADR) is a serious concern for practicing veterinarians and other health professionals, and refers to an unintended, undesired and unexpected response to a drug that negatively affects the patient's health. It may be iatrogenic or genetically induced, and may result in death of the affected animal. The ADRs are often complicated and unexpected due to myriad clinical symptoms and multiple mechanisms of drug-host interaction. Toxicity due to commonly used drugs is not uncommon when they are used injudiciously or for a prolonged period. Licosamides, exclusively prescribed against anaerobic pyoderma, often ends with diarrhoea and vomiting in canines. Treatment with Penicillin and $\beta$-lactam antibiotics induces onset of pemphigious vulgare, drug allergy or hypersensitivity. Chloroamphenicol and aminoglycosides causes Gray's baby syndrome and ototoxicity in puppies, respectively. Aminoglycosides are very often associated with nephrotoxicity, ototoxicity and neuromuscular blockage. Injudicious use of fluroquinones induces the onset of arthropathy in pups at the weight bearing joints. The most effective therapeutic measure in managing ADR is to treat the causative mediators, followed by supportive and symptomatic treatment. So, in this prospective review, we attempt to bring forth the commonly occurring adverse drug reactions, their classification, underlying mechanism, epidemiology, treatment and management as gleaned from the literature available till date and the different clinical cases observed by the authors.
\end{abstract}

Keywords: adverse drug reaction, allergy, antibiotics, canines, drug interaction, hypersensitivity, toxicity.

\section{Introduction}

The veterinarians and other health professional commonly encounter inter-individual variations in response to drug administration. Some patients respond well to a particular therapy, while others fail to demonstrate idiosyncrasy. Often, small animal clinicians are informed by the dog owners or breeders that their dogs are sensitive to a particular type of medication. This variation in drug response among individuals is due to the varied genetic make-up which explains the adverse drug reactions and offers a major step towards safe pharmacotherapy. Therefore, pharmacogenetics has been used to explain idiosyncratic drug reactions in veterinary patients. The genotypespecific approaches to therapy in childhood (GATC), a national Adverse Drug Reaction (ADR) network, has been established in Canada in 2005 to report specific ADRs in children and identify the predictive genomic biomarkers of drug risk and thus, to provide a framework of ADR surveillance [1].

Adverse drug reactions (ADRs) are associated with serious harmful effects and may lead to death in both human and animal patients. ADRs are ranked as the fifth major cause of mortality in human population

Copyright: The authors. This article is an open access article licensed under the terms of the Creative Commons Attribution License (http://creativecommons.org/licenses/by/2.0) which permits unrestricted use, distribution and reproduction in any medium, provided the work is properly cited. of the western world [1]. Around 2.9-5.6\% of all human patients admitted to hospitals suffer from ADRs and as many as $35 \%$ of them experience an ADR during their stay in hospital. The overall incidence of serious ADRs is $6.7 \%$ and of fatal ADRs is $0.32 \%$ in hospitalized patients [2]. A recent study in Turkey revealed that maximum cases on ADR in animals were reported from vitamin-mineral-amino acid preparations (39\%), followed by antibacterials and anthelmentics (26\%). The most observed adverse drug reactions were anaphylaxis and the local reactions at the injected site [3]. The documented incidence of ADR in pets is lower than that of human, probably due to fewer drugs prescribed per animal patients vis-a-vis human patients, and poor investigations on ADR in animal patients following drug administration. ADR, in many occasions, is erroneously attributed to disease progression or the onset of another disease process instead of being easily recognized [4]. The use of drugs, biological, vaccines, and dietary supplements is aimed at beneficial therapeutic outcomes, provided they are being used in accurate dose and diseases and the outcome is thoroughly monitored. However, their use is associated with inherent risks both in human and veterinary practice.

ADRs are the frequently seen problems and results in deleterious health consequences and remain a serious challenge in terms of associated morbidity and mortality [5]. Several plant-based medicinal products 
have been evaluated for ameliorating the toxic effects, even after ADR reported following administration of anticancer drug, cisplastin [6].

In this review we have detailed down the ADR associated with common antibacterials, mechanisms governing them as well as the steps involved in management and treatment. This document would serve as an overview for the veterinary clinicians and hence, prevent future injuries for companion animal practice.

\section{Adverse drug reaction}

There is no universally accepted definition for an ADR. Most definitions are more or less similar to that explained by the World Health Organization (WHO) or the United States Food and Drug Administration (USFDA). As per the WHO definition, an adverse drug event is any response to a drug that is noxious and unintended in doses normally used in people or animals for diagnosis, prevention, and treatment. On the other hand, the USFDA describes serious adverse drug events as events that result in patient's death, lifethreatening outcomes, hospitalization, disability, congenital anomaly, and outcomes requiring healthcare interventions $[1,3]$. Some ADRs may be predictable, based upon a drug's pharmacodynamic and pharmacokinetic properties or may be idiosyncratic due to underling genetic factors. Hence, an identification of predisposing genotypes may improve patient management through the prospective selection of appropriate remedy [4].

\section{Classification}

ADRs are often classified as type-A occurring frequently and typically dose related and type-B, which is often, termed as idiosyncratic reactions. Type- $\mathrm{A}$ reactions represent an extension of the drug's therapeutic effect occurring frequently, such as bleeding episodes with warfarinization [7]. In contrast, type-B reactions are unpredictable and occur only in susceptible individuals. The drug reactions can again be classified into immunologic and non-immunologic etiologies. Nearly $70-80 \%$ of ADRs are caused by predictable, non-immunologic factors. The remaining $20-25 \%$ of ADRs is unpredictable which may or may not be immune-mediated. Only 5 to $10 \%$ of these reactions constitute true drug hypersensitivity and are mostly IgE-mediated [8]. Toxic effects such as anticholinergic effects of tricyclic antidepressants, digitoxin toxicity and serotonin syndrome are dose related; whereas immunological reaction like ampicillin rash, penicillin hypersensitivity, malignant hyperthermia, and idiosyncratic reactions are not related to dose. Moreover, hypothalamic-pituitaryadrenal axis suppression following corticosteroids therapy is attributed to dose- and time related reactions. Sometimes, myocardial ischemia occurs in patients due to immediate withdrawal of $\beta$-blockers, if not withdrawn slowly. Immunological drug reactions include anaphylaxis from $\beta$-lactam antibiotic, serum sickness from anti-thymocyte globulin, contact dermatitis from topical antihistamine, hemolytic anaemia from penicillin, morbilliform rash from sulphonamides, anticonvulsant hypersensitivity syndrome, toxic epidermal necrolysis, drug-induced lupus-like syndrome [9]. Similarly, dry mouth from antihistamines, hepatotoxicity from methotrexate, thrush while taking antibiotic, seizure from theophy-lline, while taking erythromycin, and seizure from excessive lidocaine (xylocaine) are the examples of predictable non-immunologic drug reactions. Unpredictable non-immunologic drug reactions include pseudoallergic, idiosyncratic and intolerance [9].

Pseudoallergic reactions are mostly caused by direct activation of the mast cells and their degranulation by drugs such as opiates, vancomycin and radiocontrast media. The clinical signs are often same as that of type-I hypersensitivity, except that they do not involve drug specific IgE. Idiosyncratic reactions are often unpredicted and are qualitatively aberrant reactions with much less prevalence. Drug-induced hemolysis with deficiency of glucose-6-phosphate dehydrogenase (G6PD) is a good example of idiosyncratic reaction. However, drug intolerance explains a lower threshold to the normal pharmacologic action of a drug.

Presence of a foreign substance or drug or xenobiotic activate T-cells, which in turn express skin homing receptors, necessary for presentation of allergen and thus ultimately leads to cellular apoptosis and inflammation [9].

Epidemiological data support the existence of specific factors that increase the risk of ADR. Female gender, infection with human immunodeficiency virus (HIV), and herpes virus infection have been reported to increase the risk. Asthma, systemic lupus erythematosus and use of $\beta$-blockers increases the risk for drug hypersensitivity reactions [10]. Atopic patients have an increased risk for serious allergic reactions [11]. ADR have been largely confined to children. Recent studies have reported that ADR is three times higher among pediatric patients as compared to adults [12], developmentally associated with changes in drug disposition, metabolism, clearance pharmacokinetics, and pharmacodynamics $[12,13]$. Reviewing of human literature will give a lead for exploitation and further application of this knowledge in veterinary medicine. Suboptimal or inappropriate prescribing is highly prevalent in geriatric patients, and is becoming a global healthcare concern in the present worldwide elderly population demographics [14].

Veterinary dermatological patients may be particularly at risk due to necessary and frequent use of multiple drugs to achieve the desired therapeutic outcome. Treatment often involves drugs that have been implicated in adverse reactions and toxicity in other patients including antimicrobials, external and internal anti-parasitic drugs, antifungal, anti-inflammatory drugs, general anaesthetics, tranquilizers, oncolytic 
agents, vitamins, hormones, etc. There are thousands of recognized drug interactions and some may only be reported in human medicine and are not applicable to veterinary practice. On the other hand, there may be a significant number of interactions that occur only in the species we are treating in pet and companion animals. Some of the most commonly prescribed anti-microbial agents and reported to be have toxic effects in dogs are described.

a. Sulfonamides: The sulphonamides such as sulfamethoxazole, sulfadiazine, sulfadimethoxine have been reported to cause numerous dose-dependant reactions in dogs including haematuria, non-regenerative anemia, interfered thyroxine synthesis, erythema multiforme, erythema nodosum, photosensitivity, urticarial rashes and many other skin diseases [15]. The mechanism and time course of these reactions vary considerably from species to species. Anaphylaxis may not accompany the initial cutaneous drug reactions, but re-exposure can exacerbate this life-threatening reaction. These are immediate type immune-mediated reactions due to presence of IgE antibodies against the sulfonamide [15, 16]. Binding specificity studies suggest that the 5methyl-3-isoxazolyl group on sulfonamides is an active component in antibody recognition [16]. Such reactions occurring across a spectrum of sulfonamides in a given dog is suggested from in vitro cross-reactivity within a variety of sulfonamides. Fever together with a morbilliform or maculopapular, non-urticarial skin rashes are generally manifested by 7-14 days postinitiation of therapy with sulfonamide. Bio-activation of sulphonamide may result from formation of metaboliteprotein conjugates in vivo as inferred from in vitro studies demonstrating the ability of reactive metabolites of sulfonamides to bind covalently to proteins [17]. This implies that the slow acetylator phenotype patients are at a greater risk of toxicity as they bioactivate a larger fraction of the administered dose via cytochrome $\mathrm{P}_{450}$.

b. Penicillin: Penicillin and its derivatives are the common cause of ADR which vary from a morbilliform eruption to anaphylaxis $[15,18]$. Ampicillin and amoxycillin are most commonly implicated in morbilliform drug reactions. The high cutaneous reaction rate may be attributed to the diacyl side chain of these compounds that gives rise to the formation of linear polymers. Acute generalized exanthematous pustulosis is commonly caused by $\beta$-lactam and macrolide antibiotics [18]. Histological section demonstrates an intraepidermal spongiform pustule with eosinophils.

The incidence of true cross-reactivity between penicillins and cephalosporins is poorly recorded. Still, the patient's owners are often advised against fatal anaphylaxis while administering cephalosporin to pets with a history of penicillin allergy $[19,20]$. However, since 1980 the degree of cross-reactivity between penicillin and current-generation cephalosporins has been found to be less than $5 \%$ [20]. In such cases of $\beta$ - lactam allergy, penicillin skin testing is advised [21]. Carbapenem is also restricted in penicillin allergy due to the evidence of cross reactivity between other $\beta$ lactam antibiotics and penicillin $[19,22]$. In such situations, aztreonam (Azactam) is safely administered because of extremely rare cross-reactivity.

c. Cephalosporins: The penicillin and cephalosporin are $\beta$-lactam antibiotics structurally characterised by a five-membered thiazolidine ring and a six-membered dihydrothiazine ring respectively. Risk of adverse cutaneous reaction to cephalosporins in a patient with a history of penicillin allergy is controversial, but becomes visible to be quite low. Both cephalosporins and the penicillins have been implicated in inducing a pemphiguslike syndrome [18, 19,22]. The same syndrome is also induced by thiol drugs including captopril, D-penicillamine, and gold sodium thiomalate which is characterized by flaccid bullae clinically indistinguishable from pemphigus vulgaris that develop within the first few weeks of drug therapy. Direct immunofluorescence demonstrates IgG antibodies targeting the keratinocyte desmosomal components desmoglein I or III $[19,20]$. Cephalosporin also induces the onset of gastrointestinal disturbances, hemolysis and glycosuria in dogs.

d. Aminoglycosides: Renal damage and ototoxicity have been linked to repeated aminoglycoside administration or its overuse. The renal damage ranges from mild, subclinical changes to more severe nephrotoxicity and acute renal failure [23]. The animal's ability to improve depends on the nature of medication exposure and the quantity of healthy renal tissue remaining to compensate [24]. Neomycin is considered as the most nephrotoxic while aminoglycoside, dihydrostreptomycin and streptomycin are least nephrotoxic, and the other common aminoglycosides are considered somewhere intermediate between those three drugs in their toxicity [25]. Aminoglycosides cause nephrotoxicity by accumulating in the proximal tubular cells and interfere with cellular metabolism and transport processes by establishing lysosomal phospholipid complexes $[25,26]$. These tubular changes can progress to proximal tubular necrosis with increasing exposure to the drug $[26,27]$. The toxic renal changes caused by gentamicin and other aminoglycosides lessen the elimination of the antibiotic and augment serum antibiotic concentrations, thereby increasing the potential toxicity. Dogs with subclinical renal dysfunction are more sensitive to the toxicity of gentamicin and develop oliguria and acute renal failure that may not be reversible from a high gentamicin dose [24]. The local renin-angiotensin system is activated leading to local vasoconstriction causing a decrease in the glomerular filtration rate [26]. The increase in plasma creatinine $(\mathrm{sCr})$ level due to reductions in glomerular filtration rate is used as the suggestive measure of nephrotoxicity. The detectable elevation in $\mathrm{sCr}$ and/or BUN precedes mRNA expression products in urine and/or kidney that could be used as potential biomarkers for acute gentamicin related nephrotoxicity in dogs [27]. 
Nephrotoxicity is exacerbated by many other factors that compromises renal blood flow. Co-administration of certain drugs including furesomide, ethacrynic acid, cyclosporin, cisplatin, and vancomycin (but not teicoplanin) have been reported to increase the risk of nephrotoxicity [25].

Some aminoglycosides are more likely to cause auditory toxicity (cochlear otoxicity) and others are more likely to cause vestibular ototoxicity. This may be due to the distribution characteristics of each drug and its ability to concentrate in each sensory organ and result from damage to the sensory hair cells in the cochlea and the labyrinth [28]. Unlike nephrotoxicity, auditory or vestibular toxicity is often irreversible. A rare form of auditory toxicity after single dose administration of aminoglycosides has been described to be associated with two different mutations in the mitochondrial 12S ribosomal RNA gene [29].

Neuromuscular paralysis is very rare as compared to the nephrotoxic and ototoxic effects of aminoglycosides. The neuromuscular blocking effects of dihydrostreptomycin, gentamicin, neomycin, and streptomycin have been demonstrated with the dose rate of $14-43 \mathrm{mg} / \mathrm{kg}$. The postsynaptic blocking component of this effect can be reversed by a cholinesterase inhibitor, such as neostigmine, and the apparent presynapic effect can be antagonized by the administration of calcium [25].

Besides, tetracycline causes A-Colitis- $\mathrm{X}$ in horses, drug fever in cats, and photosensitivity in grazing animals.

e. Fluoroquinolones: Fluoroquinolones are an important group of antibacterial agents widely used veterinary practice in the treatment of infectious diseases [25]. It mostly causes arthropathy in young animals, retinal degeneration in cats, neuromuscular paralysis in horses. Young and growing pups are most prone to quinolones induced arthropathy especially on weight-bearing joints, the underlying mechanism of which still remains obscured [25,31]. Quinolones have an inhibitory effect on DNA, collagen and proteoglycan synthesis and on the generation of oxygen derived reactive molecules $[32,33]$. Irreversible chondrotoxicity and tendinitis associated with quinolines may also be explained on account of their chelating properties for bi- and tri-valent ions mostly magnesium ions, leading to formation of free radicals or due to an altered functionality of integrin receptors on chondrocyte and tenocytes surface [34]. Tendons cannot easily compensate these altered functions because of their poor vascularization. Apoptosis of the tendon and chondron cells, a consequence of altered $\beta$-1 integrin receptors and Map-kinase pathway is the final event in the pathogenesis of fluoroquinolone-induced tendinopathies as evidenced by cultured human tendon cell line $[32,34]$. The findings that quinolone-induced damage on connective tissue is partially due to magnesium chelation also support the observation that patients with a latent magnesium deficiency could be at an increased risk of tendon disorders [34].

A recent study has proposed a possible bilateral fluoroquinolone-associated uveitis, particularly the incidence of iris transillumination and pigment dispersion among human patients [35].

f. Chloramphenicol: Chloramphenicol, a powerful broad spectrum bacteriostatic drug, is most often administered in dogs with pneumonia, infection of the brain, eye, and in anaerobic infection. Toxic adverse effects of chloramphenicol include bone marrow depression, gastrointestinal upsets, hypersensitivity reactions and gray baby's syndrome [25, 26]. All dogs do not experience side effects while taking chloramphenicol but puppies unable to digest and process chloramphenicol the same way an adult canine can thus resulting in toxic accumulation of the chloramphenicol in the puppy's body [26]. Chloramphenicol crosses the blood brain barrier and reaches onto the foetus, and is, therefore, contraindicated in pregnant bitch. Chloramphenicol is rarely associated with blood dyscrasias where the drug targets the bone marrow [25].

Gray baby's syndrome in neonates and infants occurs due accumulation of unconjugated clroramphenicol in the body that blocks electron transport system in liver, myocardium and skeletal muscle, and is characterized by vomiting, hypothermia, ashen gray cyanosis, cardiovascular collapse and sometimes leading to death. The human and animal neonates are deficient in microsomal enzymes and inadequately metabolize the drug [26].

Several diagnostic tests and therapy are being practised to ameliorate drug hypersensitivity and ADRs. Laboratory tests including skin testing radioallergosorbent test (RAST) are conducted to study IgEmediated immune reaction with therapeutic interventions of antihistamines, systemic corticosteroids and bronchodilators. Similarly, direct or indirect Coombs' test is carried out to read the cytotoxic immune reaction. Patch test or lymphocyte proliferation assay is performed to learn the delayed and cell-mediated immune reaction and the present drug therapy is stopped with administration of antihistamines and topical or systemic corticosteroids.

g. Macrolide antibiotics: Most macrolide antibiotics do not produce serious toxic effects in animals but tylosin and tilmicosin have tendency to produce cardiovascular toxicity [26]. Very high doses and prolonged use in human and animals cause untoward effects namely, transient auditory impairment and superinfection. Oral erythromycin may be highly irritating to the stomach and when given by injection may cause severe phlebitis. The combination of some macrolides and statins used for lowering cholesterol is not advisable and can lead to debilitating myopathy [36]. This is because some macrolides (clarithromycin and erythromycin, not azithromycin) are potent inhibitors of the cytochrome $\mathrm{P}_{450}$ system, particularly of $\mathrm{CYP}_{3} \mathrm{~A}_{4}$.

h. Lincosamide antibiotics: Lincosamides are 
preferred bacteriostatic antibiotics in dermatological problems in veterinary practice. They are well absorbed orally and penetrate well into infected skin and affects the bacteria including staphylococci $[25$, 26]. Rapid development of bacterial resistance and occasional gastroenteric upset are the main disadvantages for their use [37]. Decreased milk production and ketosis are also common due to toxicity of lincosamide antibiotics in milch cows [38]. It also causes loose stools in dogs and pigs, vomition in cats, and Pseudomembranous colitis in human, horses and other herbivores respectively. However in some animals, adverse effects have been noticed with parenterally administered lincomycin.

\section{Prophylaxis and management of ADR}

An early detection of the cause of ADR and prompt treatment of anaphylaxis are essentially required for successful management. Acute therapy in emergency is directed toward oxygenation and maintenance of normotension. The most appropriate therapy includes the use of epinephrine, oxygen, vasopressors, corticosteroids and adequate fluid replacement. ADR needs to be differentiated from other symptoms to avoid the discontinuance of the necessary drugs. A complete and thorough knowledge of drugs causing immunologic reactions, patients' history of allergy if any and mastery over use of satisfactory alternatives against hypersensitivity is a must. Adverse reaction can be minimized through use of established protocols for premedication or through desensitization achieved with graduated dosage schedules.

a. Sulphonamide: A number of sulphonamides and their combinations are being used to treat respiratory, urinary tract, skin, or gastrointestinal infections in veterinary practice. However, it is contraindicated in pregnant or nursing animals, dehydrated animals and in those animals suffering from bladder or kidney stones, liver or kidney diseases. The pet should be encouraged to drink plenty of water to prevent crystalluria formation in the kidneys. Similarly, adequate drinking water should be provided to dogs with routine monitoring of blood cell abnormalities during sulphonamide administration.

b. Penicillin: Penicillin for dogs is prescribed for infections of the urinary tract, lungs, and kidneys. Resistance to it mostly encountered when the drug administration is stopped before the prescribed course is completed, thus prompting a chance for the infection to return. A topical penicillin lotion or ointment may be prescribed for various skin infections in dogs and also sometimes used in treating leptospirosis, wounds, and dental infections. In dogs and cats, penicillin- $\mathrm{V}$ is administered@5.5-11mg/kg b wt at every 6-8 hour interval. [25, 39].It is not used in animals hypersensitive (allergic) to penicillins or $\beta$-lactam antibiotics such as cephalosporins. Penicillins can cross the placenta, and hence, is not advised in pregnant animals although no detrimental results to fetuses have been reported.

c. Cephalosporins: Cephalosporins are broad spectrum bactericidal antibiotics used to treat bacterial infections of the skin, urinary tract, respiratory tract, bones and joints $[25,26,39]$. Cefadroxil is the most commonly prescribed and FDA approved medication used to treat dogs and cats. However the drug is used with much caution in pregnant and nursing animals as well as in combination with probenecid or aminoglycosides which may aggravate neuromuscular blockade or nephrotoxicity $[25,40]$.

d. Aminoglycosides: Aminoglycosides are not used in animals hypersensitive (allergic) to aminoglycosides and are also not prescribed in anaerobic bacterial infection. Aminoglycosides is contraindicated in dogs used for hearing sensation very frequently to perform their work as the vestibular impairment is often irreversible. Gentamicin therapy is also contraindicated in nephrotoxicity, neuromuscular disorders and in pregnancy or nursing animals $[32,37,38]$.

Renal damage in older dogs and heartworm microfilaria infection, hypovolemic dehydration are often the predisposing factors for aminoglycoside toxicity [40]. It is highly advised to monitor the renal function during the course of treatment mostly through urinalysis indicate early nephrotoxicity. The nephrotoxicity of aminoglycoside is checked by adjusting the drug dosing rate and duration of therapy, which under no circumstance should be more than the recommended dose in plasma [40, 41]. It is always commended to avoid concurrent or sequential use of nephrotoxic, ototoxic, or neuromuscular blocking drugs, particularly other aminoglycosides because of their additive effects. Concurrent administration of furosemide with aminoglycosides may enhance nephrotoxicity $[25,26$, 39].

Diuretics should not be employed together with aminoglycosides as it increases the risk of kidney damage. Products causing hearing loss, vestibular disease, or kidney disease should not be recommended along with aminoglycoside therapy in order to shy off further complications [40, 41]. The possibility of neuromuscular blockade and respiratory paralysis exists if aminoglycosides are given to patients receiving anaesthetics, neuromuscular blockers, or massive transfusions of citrate-anticoagulated blood. Calcium salts may reverse neuromuscular blockade if it occurs.

\section{Conclusion}

ADR or drug toxicity is a common problem that threatens the safety and health status of the patients, but as the whole represents burden on the whole health care system. It is often poorly diagnosed and documented in day to day medical practice. The situation is even grimmer in veterinary field conditions particularly when prescribed by quacks, frequent practice of polypharmacy or fixed dose combinations. The diagnosis of ADR and mechanisms involved are also often hardly studied and 
understood by the unskilled practitioners, and they attribute these symptoms to the progress of the disease. Such scenario is more frequently India, particularly in the companion animal practices, where over the counter prescription is common and door step services are being provided by personnel without having a valid registration ID from Veterinary Council of India or State Veterinary Council.

Non-reporting of ADR encountered by the clinician is the biggest disadvantage to curb this situation. The health practioners are required to devote more attention to ADR and to identify risk factors in suspected idiosyncratic ADRs [42].

The first step in managing ADR or toxicity is to discontinue the causative medication and its substitution with alternative medications having unrelated chemical structures. The clinical consequences of cessation of medication or substitution need to be closely monitored by a competent professional. In majority of patients, symptoms usually resolve within two weeks. Systemic corticosteroids speed up the recovery and use of topical corticosteroids and oral antihistamines improve the dermatologic symptoms. Elucidation of the key players in mediation of ADRs will provide therapeutic targets for its management and prevention.

\section{Authors' contributions}

KA and IM prepared the initial version of the manuscript. GS and SP assisted in literature collection and edited the final manuscript. IM, KKS and RCP drafted and revised the manuscript for critical scientific corrections. All authors read and approved the final manuscript.

\section{Acknowledgements}

The authors are thankful to e-Library section of Central Library, Orissa University of Agriculture \& Technology, Bhubaneswar for providing scientific literature.

\section{Competing interests}

The authors declare that they have no competing interests.

\section{References}

1. Carleton B., Poole R., Smith M., Leeder J., Ghannadan R., Ross C., Phillips M. and Hayden, M. (2009) Adverse drug reaction active surveillance: developing a national network in Canada's children's hospitals, Pharmacoepidem. Drug Saf., 18: 713-721.

2. Singh A. and Bhatt P. (2012) Comparative evaluation of adverse drug reaction reporting forms for introduction of a spontaneous generic ADR form, J. Pharmacol. Pharmacother, 3(3): 228-232.

3. Dogan F. (2011) Pharmacovigilance in veterinary profession, Euras. J. Vet. Sci., 27(1): 19-25.

4. Mealey K.L. (2004) Clinically significant drug interactions. $19^{\text {th }}$ Proceedings of AAVD \& ACVD annual meeting (North American Dermatology Forum). p45-49.

5. Lopez-Gonzalez E., Herdeiro M.T. and Figueiras, A. (2009) Determinants of Under-Reporting of Adverse Drug Reactions, Drug Saf., 32(1): 19-31.

6. Ramya B., Anjaneyulu Y., Gopala Reddy A., Madhuri D., Lakshman M. and Shivakumar, P. (2013) Protective role of turmeric on histological, ultrastructural and serobiochemical changes in cisplatin-induced nephrotoxicity in female rats, Vet. World, 6: 865-869.

7. Naisbitt D.J., Gordon S.F., Pirmohamed M. and Park, B.K. (2000) Immunological principles of adverse drug reactions: the initiation and propagation of immune responses elicited by drug treatment, Drug Saf., 23: 483-507.

8. DeShazo R.D. and Kemp S.F. (1997) Allergic reactions to drugs and biologic agents, J. Am. Med. Assoc., 278: 18951906.

9. Roychowdhury S. and Svensson C.K. (2005) Mechanisms of Drug-induced Delayed-type Hypersensitivity Reactions in the Skin, Am. Assoc. Pharmac. Sci. J., 7(4): E834-E846.

10. Barranco P. and Lopez-Serrano M.C. (1998) General and epidemiological aspects of allergic drug reactions, Clin. Exp. Allergy, 28(4): 61-2.

11. Adkinson N.F. (1984) Jr. Risk factors for drug allergy, $J$. Allergy Clin. Immunol., 74: 567-72.

12. Du W., Lehr V.T., Lieh-Lai M., Koo W., Ward R.M., Rieder M.J., Van Den Anker J.N., Reeves J.H., Mathew M., LulicBotica M. and Aranda, J.V. (2013) An Algorithm to Detect Adverse Drug Reactions in the Neonatal Intensive Care Unit, J. Clin. Pharma., 53: 87-95.

13. Du W., Tutag Lehr, V., Caverly M., Kelm L., Reeves J. and Lieh-Lai M. (2013) Incidence and Costs of Adverse Drug Reactions in a Tertiary Care Pediatric Intensive Care Unit, $J$. Clin. Pharma., 53: 567-573.

14. Hamilton H.J., Gallagher P.F. and O'Mahony D. (2009) Inappropriate prescribing and adverse drug events in older people, BMC Geriatrics, 9: 5-8.

15. Miller W.H., Griffin C.E. and Campbell, K.L. (2013) Bacterial Skin Diseases. In: Miller W.H., Griffin C.E. and Campbell K.L. editor. Muller and Kirk's Small Animal Dermatology. 7th edition. Elsevier, St. Louis, Missouri, USA. p191-192.

16. Harle D.G., Baldo, B.A. and Wells J.V. (1988) Drugs as allergens: detection and combining site specificities of $\operatorname{IgE}$ antibodies to sulfamethoxazole, Mol. Immunol., 25: 1347-1354.

17. Brackett C.C., Singh H. and Block J.H. (2004) Likelihood and Mechanisms of Cross-Allergenicity Between Sulfonamide Antibiotics and Other Drugs Containing a Sulfonamide Functional Group, Pharmacotherapy, 24: 856-870.

18. Voie K.L., Lucas B.E., Schaeffer D., Kim D., Campbell K.L. and Lavergne S.N. (2013) The effect of 'allergenic' and 'nonallergenic' antibiotics on dog keratinocyte viability in vitro, Vet. Dermatol., 24: 501-e119.

19. Romano A., Gaeta F., Valluzzi R.L., Caruso C., Rumi G. and Bousquet P.J. (2010) IgE-mediated hypersensitivity to cephalosporins: Cross-reactivity and tolerability of penicillins, monobactams, and carbapenems, J. Allergy Clin. Immunol., 126(5): 994-999.

20. James C.W. and Gurk-Turner C. (2001) Cross-reactivity of beta-lactam antibiotics, BUMC Proceed, 14: 106-107.

21. Fox S. and Park M.A. (2011) Penicillin skin testing in the evaluation and management of penicillin allergy, Annals of Allergy, Asthma \& Immunol., 106 (1): 1-7.

22. Crowson A.N. and Magro C.M. (1999) Recent advances in the pathology of cutaneous drug eruptions, Dermatol. Clin, 17: 537-560.

23. Brown S.A. and Garry, F.B. (1988) Comparison of serum and renal gentamicin concentrations with fractional urinary excretion tests as indicators of nephrotoxicity, $J$. Vet. Pharmacol. Ther, 11(4): 330-7.

24. Frazier D.L., Aucoin D.P. and Riviere J.E. (1988) Gentamicin pharmacokinetics and nephrotoxicity in naturally acquired and experimentally induced disease in dogs, J. Am. Vet. Med. Assoc, 192(1): 57-63.

25. Sandhu H.S. and Rampal S. (2006) Essentials of Veterinary Pharmacology and Therapeutics. 1st Edition. Kalyani, New Delhi.p1093-1186.

26. Hsu W.H. and Ahrens F.A. (2013) Drug Interactions and 
Adverse Drug Reactions. Handbook of Veterinary Pharmacology. Wiley Publishers. p461-470.

27. McDuffie J.E., Gao J., Ma J., La D., Bittner A., Sonee M., Wagoner M. and Snook S. (2013) Novel genomic biomarkers for acute gentamicin nephrotoxicity in dog, Open J. Mol. Integr. Physiol., 3: 125-133.

28. Xie J., Talaska A.E. and Schacht J. (2011) New developments in aminoglycoside therapy and ototoxicity, Hearing Res., 281:28-37.

29. Fischel-Ghodsian N. (1999) Genetic factors in aminoglycoside toxicity, Ann. N. Y. Acad. Sci, 884:99-109.

30. Sykes J.E. and Papich M.G. (2014) Antibacterial Drugs, Canine and Feline Infectious Diseases, Saunders, W.B., p6686.

31. Stahlmann R. and Hartmut L. (2010) Safety Considerations of Fluoroquinolones in the Elderly, Drugs Aging, 27(3): 193-209.

32. Simonin M.A., Gegout-Pottie P., Minn A., Gillet P., Netter P. and Terlain, B. (1999) Proteoglycan and collagen biochemical variations during fluoroquinolone- induced chondrotoxicity in mice, Antimicrob. Agents Chemother, 43: 2915-2921.

33. Hall M.M., Finnoff J.T. and Smith J. (2011) Musculoskeletal Complications of Fluoroquinolones: Guidelines and Precautions for Usage in the Athletic Population, Phys. Med. Rehabil., 3(2): 132-142.

34. Stahlmann R., Kuhner S., Shakibaei M., Flores J., Vormann J. and van Sickle D.C. (2000) Effects of magnesium deficiency on joint cartilage in immature beagle dogs: immunohistochemistry, electron microscopy, and mineral concentrations, Arch. Toxicol., 73:573-580.

35. Hinkle D.M., Dacey M.S., Mandelcorn E., Kalyani P., Mauro J., Bates J.H., Soukasian S.H., Holland G.N., Foster C.S., Fraunfelder F.T., Davis J.L. and Fraunfelder F.W. (2012) Bilateral uveitis associated with fluoroquinolone therapy, Cutaneous Ocul Toxicol., 31(2): 111-116.

36. Sathasivam S. and Lecky B. (2008) Statin induced myopathy, Brit. Med. J., 337: 2286.

37. Noli C. and Boothe D. (1999) Macrolides and lincosamides, Vet. Dermatol., 10:217-223.

38. Rice D.A. and McMurray C.H. (1983) Ketosis in dairy cows caused by low levels of lincomycin in concentrated feed, Vet. Rec., 113:495-6.

39. Richard A.H. (2001) Veterinary Pharmacology and Therapeutics. 8th edition. Iowa State University: Blackwell, Iowa, U.S.A., p844.

40. Giguère S., Prescott J.F. and Dowling P.M. (2013) Antimicrobial Therapy in Veterinary Medicine. 5th edition. John Wiley \& Sons, Iowa State University.,p67.

41. Prayle A., Watson A.A., Fortnum H. and Smyth A. (2010) Side effects of aminoglycosides on the kidney, ear and balance in cystic fibrosis, Thorax, 65(7): 654-658.

42. Trumic E., Pranjic N., Begic L., Becic F. and Asceric M. (2012) Idiosyncratic Adverse Reactions of Most Frequent Drug Combinations Longterm Use Among Hospitalized patients with Polypharmacy, Med.Arch., 66 (4): 243-248. 